\title{
MicroRNA.320 inhibits cell proliferation and invasion in breast cancer cells by targeting SOX4
}

\author{
JUN-WEN BAI ${ }^{1-5^{*}}$, XIA WANG $^{5 *}$, YA-FENG ZHANG ${ }^{5}$, GUO-DONG YAO 5 and HONG LIU ${ }^{1,4}$ \\ ${ }^{1}$ The Second Department of Breast Cancer; ${ }^{2}$ Key Laboratory of Cancer Prevention and Therapy; ${ }^{3}$ Key Laboratory of Breast \\ Cancer Prevention and Therapy, Tianjin Medical University Cancer Institute and Hospital, National Clinical Research \\ Center for Cancer; ${ }^{4}$ Tianjin's Clinical Research Center for Cancer, Tianjin 300060; ${ }^{5}$ Department of Surgery, Affiliated \\ Hospital of Inner Mongolia Medical University, Huhhot, Inner Mongolia Autonomous Region 010050, P.R. China
}

Received May 15, 2016; Accepted August 1, 2017

DOI: $10.3892 / \mathrm{ol} .2017 .7087$

\begin{abstract}
Dysregulation of microRNAs (miRs) can contribute to cancer development and progression. In the present study, the function and underlying molecular mechanisms of miR-320 in breast cancer tumorigenesis and progression were investigated. The results of a reverse transcription-quantitative polymerase chain reaction analysis demonstrated that miR-320 was frequently downregulated in breast cancer tissues compared with adjacent normal tissues. In addition, knockdown of miR-320 in breast cancer cell lines promoted cell proliferation and invasion in vitro, whereas miR-320 overexpression had the opposite effect. Furthermore, a Dual-Luciferase reporter assay indicated that SRY-box 4 (SOX4) is a direct target of miR-320, and the restoration of SOX4 in miR-320-overexpressing cells attenuated the tumor-suppressive effects of miR-320. Collectively, these results indicated that miR-320 acts as a tumor suppressor in breast cancer tumorigenesis and progression.
\end{abstract}

\section{Introduction}

Breast cancer is the most commonly diagnosed type of cancer and the second leading cause of cancer-associated mortality in females worldwide, accounting for $29 \%$ of the total new cancer cases and $14 \%$ of the total cancer-associated mortalities in 2016 (1). A previous study demonstrated that various proteins are dysregulated in primary tumors, and are associated with breast cancer development and progression (2). Therefore, an improved understanding of the functions and the underlying molecular mechanisms of these proteins, particularly in

Correspondence to: Professor Hong Liu, The Second Department of Breast Cancer, Tianjin Medical University Cancer Institute and Hospital, National Clinical Research Center for Cancer, Huanhuxi Road, Hexi, Tianjin 300060, P.R. China

E-mail: lhbai2014@163.com

*Contributed equally

Key words: microRNA 320, SRY-box 4, epithelial-mesenchymal transition, breast cancer epithelial-mesenchymal transition, may provide novel insights into the breast tumorigenesis and progression, and enable the development of effective anti-cancer therapeutics.

The SRY-box 4 (SOX4) gene, which is located on chromosome $6 \mathrm{p} 22.3$, encodes a $47-\mathrm{kDa}$ protein that is a member of the sex-determining region Y-related high-mobility group-box transcription factor family and has functions in embryonic development and cell differentiation $(3,4)$. SOX4 has been demonstrated to be upregulated in a number of types of cancer, and increased SOX4 activity was found to contribute to cancer development and progression (5-8). SOX4 has been revealed to induce EMT, a key process during organ development and the progression of epithelial tumors to metastatic cancers (9), via its activation of the Wnt and transforming growth factor $\beta$ signaling pathways in cancer cells $(10,11)$. Previously, it has been demonstrated that certain transcriptional targets of SOX4 are associated with cancer development and progression and the processing of microRNAs (miRNAs/miRs) (12).

miRNAs are small non-coding RNAs ( 22 nucleotides in length) that regulate the expression of their target genes by translational repression or mRNA degradation $(13,14)$. miRNAs participate in crucial biological processes, including development, differentiation and proliferation $(15,16)$. Previous studies have suggested that miRNAs are involved in a number of types of cancer $(17,18)$. Differential expression of miRNAs has been widely described in breast cancers, and suggests that certain miRNAs, including miR-206 (19), miR-129 (20), miR-200 (21) and miR-34 (22), may function as oncogenes or tumor suppressors in breast cancer. Therefore, miRNAs are now considered to be important in the development of biomarkers, and may be targets for the diagnosis and treatment of breast cancer patients (23).

To understand the molecular mechanism of SOX4 in breast cancer development and progression, the present study aimed to identify miRNAs that regulate the expression of SOX4, which revealed miR-320 as a potential candidate. It has been previously identified that miR-320 is significantly downregulated, and can inhibit proliferation, metastasis and angiogenesis, in a number of types of cancer, including cervical cancer, colon cancer, glioma and prostate cancer; this evidence suggests that miR-320 functions as a tumor suppressor (24-29). However, the function and molecular mechanism of miR-320 remain unknown. 
In the present study, miR-320 was demonstrated to be frequently downregulated in breast cancer tissues compared with adjacent normal tissues. Furthermore, SOX4 was confirmed as a direct target of miR-320, and miR-320 was shown to suppress breast cancer development and progression via the downregulation of SOX4. The results of the present study indicate that miR-320 overexpression may represent a novel therapeutic strategy for patients with breast cancer.

\section{Materials and methods}

Cell culture and tissue specimens. The human breast cancer cell lines MCF7, T47D, MDA-MB-231, MDA-MB-468 and 293FT were purchased from the American Type Culture Collection (Manassas, VA, USA). MDA-MB-231 and MDA-MB-468 cells were maintained in Leibovitz's L15 medium (Gibco; Thermo Fisher Scientific, Inc., Waltham, MA, USA), and 293FT, MCF7 and T47D cells were maintained in Dulbecco's modified Eagle's medium (Gibco; Thermo Fisher Scientific, Inc.). All cell lines were supplemented with $10 \%$ fetal bovine serum (FBS), as well as penicillin and streptomycin, and were incubated at $37^{\circ} \mathrm{C}$ in a humidified atmosphere containing $5 \% \mathrm{CO}_{2}$.

In addition, primary breast cancer tissues and paired adjacent normal breast tissues were obtained from 15 females aged between 40 to 65 years (mean age, 53 years) who underwent breast surgery in the Affiliated Hospital of Inner Mongolia Medical University between April 2015 and December in 2015. All patients were diagnosed with invasive ductal carcinoma according to the morphologic criteria described in the World Health Organization histologic classification of tumors of the breast (30). Of the 15 patients, 5 patients were stage II and 10 patients were stage I. All samples were evaluated and subject to histological diagnosis by pathologists. All protocols in the present study were approved by the ethics committee of the Affiliated Hospital of Inner Mongolia Medical University, and all patients provided written informed consent.

Reverse transcription-quantitative polymerase chain reaction $(R T-q P C R)$. Total RNA was extracted from the breast cancer tissues and cell lines using TRIzol reagent (Invitrogen; Thermo Fisher Scientific, Inc.) following the manufacturer's protocol. A total of $2 \mu \mathrm{g}$ RNA was used for RT-qPCR with the Qiagen OneStep RT-PCR kit (Qiagen Benelux BV, Venlo, The Netherlands) in the following conditions: $37^{\circ} \mathrm{C}$ for $30 \mathrm{~min}$, $85^{\circ} \mathrm{C}$ for $5 \mathrm{sec}$ and kept at $4^{\circ} \mathrm{C}$ until use. TaqMan microRNA assays (Applied Biosystems; Thermo Fisher Scientific, Inc.) were performed to quantify the relative expression of miR-320 using the following thermocycling conditions: $95^{\circ} \mathrm{C}$ for $3 \mathrm{~min}, 95^{\circ} \mathrm{C}$ for $15 \mathrm{sec}$ and $60^{\circ} \mathrm{C}$ for $30 \mathrm{sec}$ for a total of 40 cycles, as previously described (31). U6 was used as the normalization control. The following primers were used: miR-320 forward, 5'-ACACTCCAGCTGGGAAAAGCT GGGTTGAGA-3'; miR-320 reverse, 5'-TGGTGTCGTGGA GTCG-3; U6 forward, 5'-CTCGCTTCGGCAGCACA-3'; U6 reverse, 5'-AACGCTTCACGAATTTGCGT-3'; GAPDH forward, 5'-AATCCCATCACCATCTTCCA-3; GAPDH reverse, 5'-CCTGCTTCACCACCTTCTTG-3; SOX4 forward, 5'-ACCGGGACCTGGATTTTAAC-3'; and SOX4 reverse, 5'-AAACCAGGTTGGAGATGCTG-3'. The CT value of GAPDH or U6 was subtracted from the CT value of target genes to obtain $\triangle \mathrm{CT}$. The relative expression level of miR-320 and SOX4 was determined as $2^{-\Delta \mathrm{CT}}$ as previously described (31). Three independent experiments were conducted.

Transfection. MDA-MB-231 or MCF7 cells were seeded in 6 -well plates at a density of $5 \times 10^{6}$ cells/well. The cells in each well were transfected with a solution of $3 \mu \mathrm{g}$ pcDNA3.1-SOX4 (GenePharma Co., Ltd., Shanghai, China) or 20 nM RNA [miRNA-320 mimics, 5'-AAAAGCUGGGUUGAGAGGGCG A-3'; inhibitors, 5'-CCUCUCAACCCAGCUUUU-3'; negative control (NC)/mimic control, 5'-UUCUCCGAACGUGUCACG UTT-3'; and inhibitor control, 5'-CAGUACUUUUGUGUA GUACAA-3'; GenePharma Co., Ltd.] using Lipofectamine 3000 (Invitrogen; Thermo Fisher Scientific, Inc.) according to the manufacturer's protocol. Subsequent experiments were conducted $24 \mathrm{~h}$ after transfection.

Cell proliferation assays. MTT and colony formation assays were used to evaluate cell proliferation. In brief, for the MTT assay (Sigma-Aldrich; Merck KGaA, Darmstadt, Germany), a total of $5 \times 10^{3}$ MDA-MB-231 or MCF7 cells/well were seeded in 96-well plates, and MTT was added to a final concentration of $0.5 \mathrm{mg} / \mathrm{ml}$, prior to the incubation of cells at $37^{\circ} \mathrm{C}$ for $4 \mathrm{~h}$. Following the removal of the culture medium, $150 \mu 1$ dimethyl sulfoxide was added and the absorbance at $570 \mathrm{~nm}$ was measured using a microplate reader. The mimic or inhibitor control transfected cells were as the negative control group.

For colony formation assay, $1 \times 10^{3} /$ well MDA-MB-231 or MCF7 cells were seeded into $6 \mathrm{~cm}$ dish after transfection. After 2 weeks of culture, the cells were stained with $1 \%$ crystal violet for $15 \mathrm{~min}$ at room temperature, after fixation with $10 \%$ formaldehyde for $30 \mathrm{~min}$ at room temperature.

Transwell invasion assay. Transwell Matrigel-coated chambers (BD Biosciences, Franklin Lakes, NJ, USA) were used to determine cell invasion. A total of $1 \times 10^{5} /$ well cells transfected with miR-320 mimics, inhibitors or NC were suspended in $200 \mu \mathrm{l}$ medium containing $1 \%$ FBS, and seeded on the upper chamber. A total of $600 \mu \mathrm{l}$ medium containing $10 \% \mathrm{FBS}$ was added to the lower chamber. Following incubation for $24 \mathrm{~h}$, cells that remained in the upper chamber were removed and the migrated cells were fixed in methanol, and subsequently stained with crystal violet for $30 \mathrm{~min}$ at room temperature. Cells were counted in five randomly selected fields using a light microscope. The mimic or inhibitor control transfected cells were as the negative control group. The cells were counted in 5 randomly selected microscopic fields (magnification, $x 400$ ) from each chamber.

Cell cycle and apoptosis assays. To determine cell cycle distribution, $1 \times 10^{6} /$ well MDA-MB-231 or MCF7 cells were collected by trypsinization and fixed in ice-cold $70 \%$ ethanol overnight. Subsequently, cells were washed with PBS and incubated with propidium iodide (PI; Sigma-Aldrich; Merck $\mathrm{KGaA}$ ) containing RNase for $30 \mathrm{~min}$ at room temperature. The cell cycle distribution was analyzed using the FACScan flow cytometer (BD Biosciences). For the apoptosis assay, 1x10\% $/$ well MDA-MB-231 cells were harvested and double-stained with annexin V-fluorescein isothiocyanate (FITC) and PI by using 
the annexin V-FITC Apoptosis Detection kit (BD Biosciences) following the manufacturer's protocol. Subsequently, each sample was analyzed using the FACScan flow cytometer. The data were processed using the ModFit LT 3.2 software (Verity Software House, Inc., Topsham, ME, USA).

Western blot analysis. Cells were harvested using cell scrapers, washed with PBS and lysed using RIPA lysis buffer (Beyotime Institute of Biotechnology, Haimen, China) with a protease inhibitor cocktail (Pierce; Thermo Fisher Scientific, Inc.) and centrifuged at $10,000 \mathrm{x}$ g for $15 \mathrm{~min}$ at $4^{\circ} \mathrm{C}$ to remove cellular debris. Protein samples $(50 \mu \mathrm{g})$ were separated using SDS-PAGE (10\% gels) and subsequently transferred to a polyvinylidene fluoride membrane (Millipore; Merck KGaA). The membranes were blocked in 5\% non-fat milk for $1 \mathrm{~h}$ and incubated at $4{ }^{\circ} \mathrm{C}$ overnight with the following primary antibodies: Anti-GAPDH (catalog no. 2118; dilution, 1:2,000; Cell Signaling Technology, Inc., Danvers, MA, USA), anti-SOX4 (catalog no. ab86809; dilution, 1:1,000; Abcam, Cambridge, MA, USA), anti-epithelial (E-)cadherin (catalog no. sc-59780; dilution, 1:1,000; Santa Cruz Biotechnology, Inc., Dallas, TX, USA) and anti-vimentin (catalog no. sc-66002; dilution, 1:2,000; Santa Cruz Biotechnology, Inc.). Membranes were washed three times with Tris-buffered Saline-Tween 20 and incubated for $1 \mathrm{~h}$ with an anti-rabbit horseradish peroxidase (HRP)-conjugated secondary antibody (catalog no. 7074; dilution, 1:2,500; Cell Signaling Technology, Inc.) or an anti-mouse HRP-conjugated secondary antibody (catalog no. 7076; dilution, 1:2,500; Cell Signaling Technology, Inc.) at room temperature. Specific proteins were detected using an ECL kit (Millipore; Merck KGaA).

Bioinformatic prediction of miR-320 potential targets. The microRNA.org targets and expression (www.microrna.org) database was used to predict potential targets for miR-320, as previously described (32).

Luciferase reporter assay. The SOX4 3'-untranslated region (UTR) containing the wild-type (wt) or mutated form of the miR-320 binding site were cloned into the psiCHECK2 vector (Promega Corporation, Madison, WI, USA). A total of $2 \times 10^{5} /$ well $293 \mathrm{FT}$ cells, cultured in 12 -well plates, were co-transfected using Lipofectamine 3000 with psiCHECK2 vectors containing either the wt or mutated SOX4 3'-UTR fragments and the control vector, as well as the miR-320 mimics and NC. Luciferase assays were performed $48 \mathrm{~h}$ after transfection, using the Dual-Luciferase Reporter Assay kit (Promega Corporation), according to the manufacturer's protocol. Firefly luciferase activity was normalized to Renilla luciferase activity.

Statistical analysis. Statistical analysis was performed using SPSS software (version 22.0; IBM Corp., Armonk, NY, USA). The Student's t-test was used to perform comparisons between two groups of data. Multiple comparisons between data were performed using a one-way analysis of variance, followed by Dunnett's test. The results were expressed as the mean \pm standard error. $\mathrm{P}<0.05$ was considered to indicate a statistically significant difference. Three independent experiments were conducted.

\section{Results}

miR-320 expression is frequently downregulated in breast cancer. RT-qPCR was used to determine the expression levels of miR-320 in the breast cancer cell lines MCF7, T47D, MDA-MB-231 and MDA-MB-468. The results demonstrated that miR-320 is expressed mostly highly in MCF7 and T47D cells, and is expressed at a relatively decreased level in MDA-MB-231 and MDA-MB-468 cells (Fig. 1A). Subsequently, the expression of miR-320 was analyzed using RT-qPCR in primary breast cancer tissues and paired adjacent normal breast tissues from 15 patients. In 12 of the 15 cases (80\%), the expression of miR-320 was downregulated in the breast cancer tissue compared with the corresponding adjacent normal tissue $(\mathrm{P}<0.001$; Fig. 1B).

miR-320 inhibits the proliferative and invasive abilities of breast cancer cells. MDA-MB-231 cells were transfected with miR-320 mimics or NC oligonucleotides and the transfection efficiency was examined by RT-qPCR. Using RT-qPCR, it was validated that miR-320 expression level was increased by miR-320 mimic in MDA-MB-231 cells (Fig. 2A). Colony formation and MTT assays revealed that overexpression of miR-320 inhibited the cell proliferation of MDA-MB-231 cells (Fig. 2B and C). Furthermore, the cell cycle distribution assay demonstrated that there was an accumulation in the G0/G1 phase among miR-320-overexpressing MDA-MB-231 cells compared with NC cells (Fig. 2D). The apoptosis assay revealed that the miR-320-overexpressing MDA-MB-231 cells exhibited an increase in the apoptotic index compared with that of control cells (Fig. 2E). To investigate the function of miR-320 in breast cancer progression, the Transwell invasion assay was performed in transfected MDA-MB-231 cells. The results demonstrated that the overexpression of miR-320 inhibited breast cancer cell invasion compared with the NC (Fig. 2F). These results suggested that miR-320 suppresses breast cancer progression.

Inhibition of miR-320 promotes breast cancer proliferation and invasion. The expression of miR-320 in MCF7 cells was knocked down by transfection of an miR-320 inhibitor or NC (Fig. 3A). The MTT and colony formation assays revealed that knockdown of miR-320 expression promoted cell proliferation in MCF7 cells compared with that in the NC group (Fig. 3B and C). The cell cycle distribution assay demonstrated that there was an accumulation of S-phase cells among the miR-320-depleted MCF7 cells compared with the NC cells (Fig. 3D). Furthermore, the Transwell invasion assay demonstrated that the number of invaded cells was increased in miR-320-depleted MCF7 cells compared with the NC group (Fig. 3E). Thus, these results indicate that inhibition of miR-320 promotes breast cancer progression.

SOX4 is a target of miR-320. SOX4 was predicted as a potential target of miR-320 using the online database microRNA.org (Fig. 4A). The online database identified two potential miR-320 binding sites on the SOX4 3'-UTR, so two different reporter plasmids were constructed (SOX4-1wt and SOX4-2wt). The SOX4 3'-UTR was cloned into a luciferase reporter vector and the putative miR-320 binding site was mutated using a 

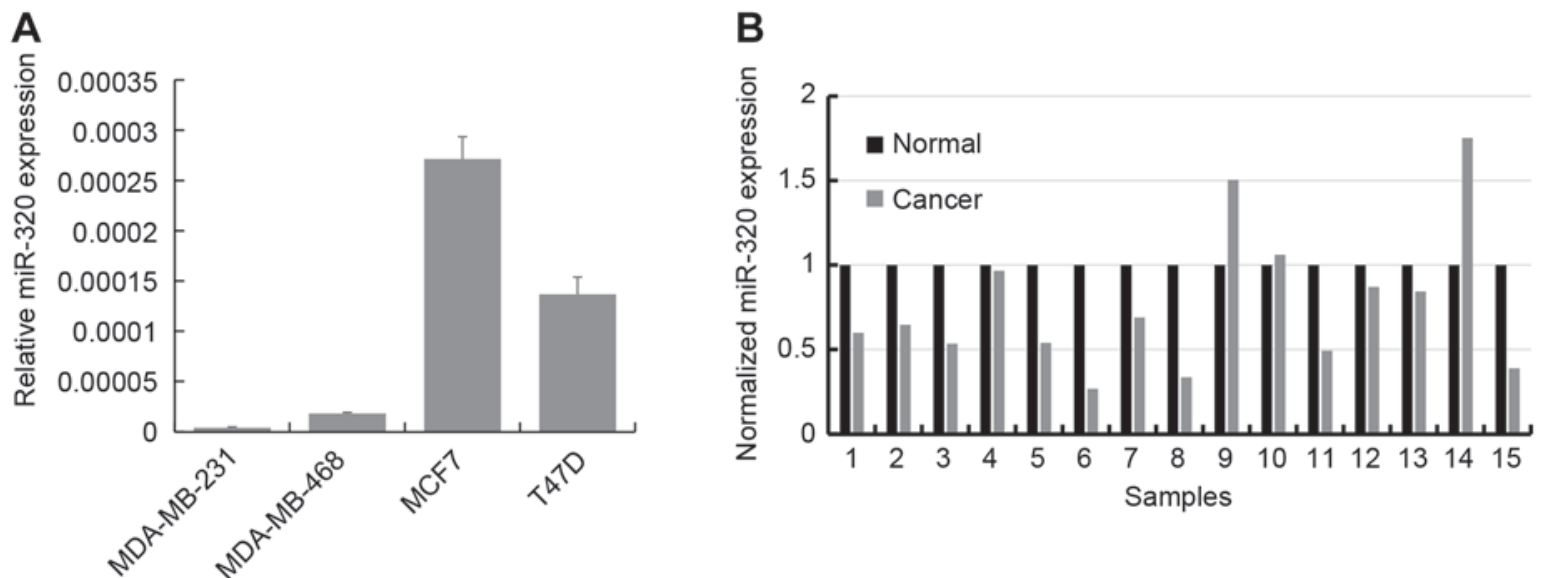

Figure 1. The expression of miR-320 is decreased in breast cancer cell lines and tissues. (A) Expression of miR-320 in four different breast cancer cell lines, determined using RT-qPCR. (B) Expression of miR-320 in breast cancer tissues and the corresponding adjacent normal breast tissue, determined using RT-qPCR. miR, microRNA; RT-qPCR, reverse transcription-quantitative polymerase chain reaction.

A
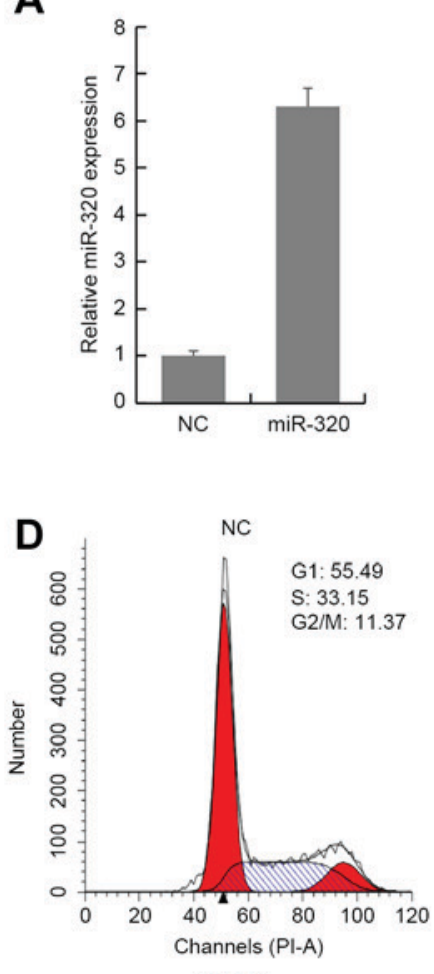

miR-320

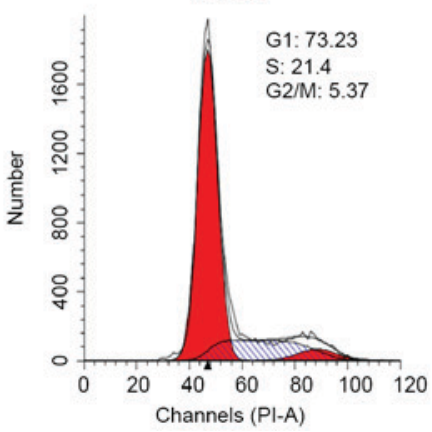

B

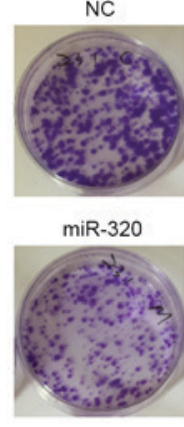

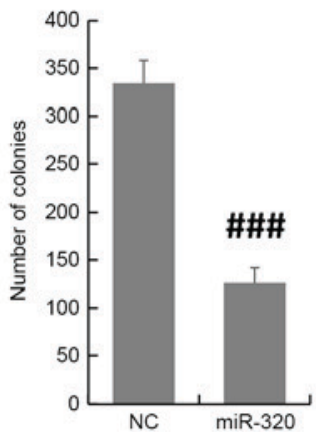

C

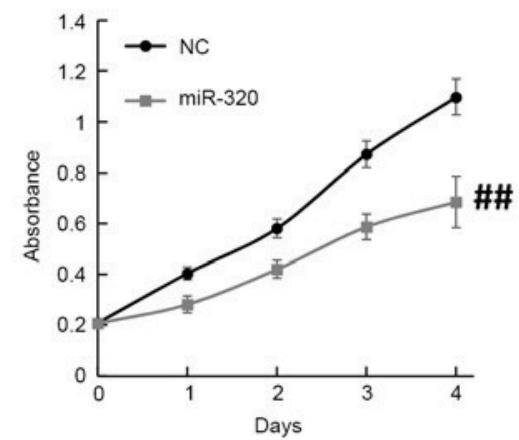

E
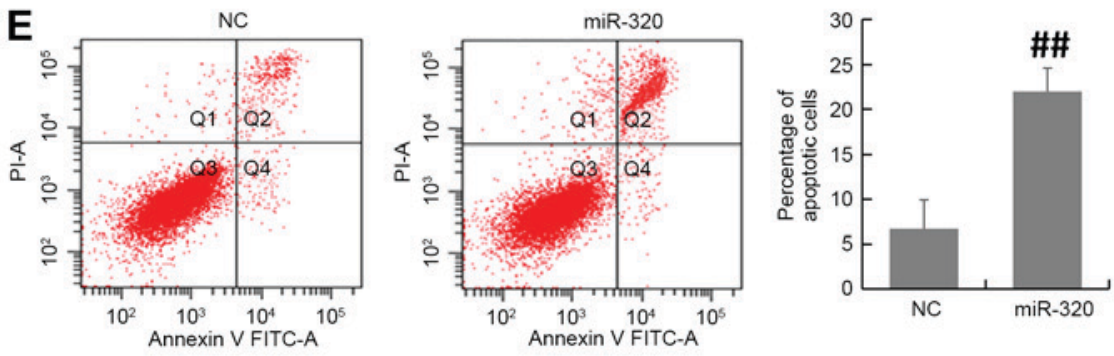

$\mathbf{F}$
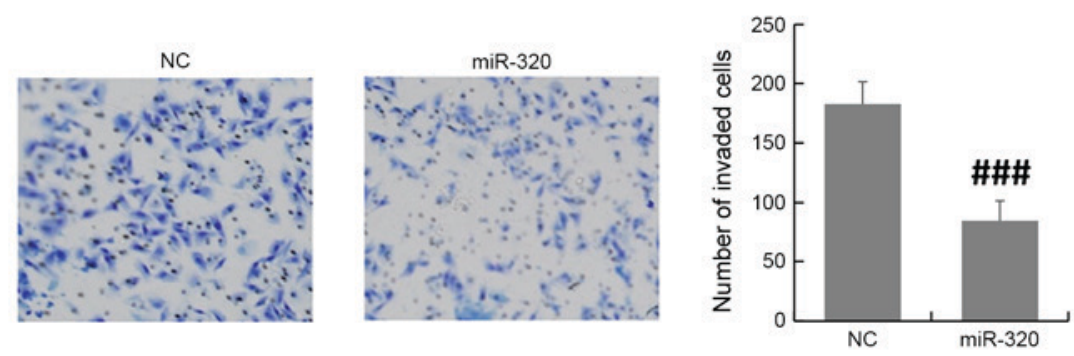

Figure 2. Overexpression of miR-320 inhibits cell proliferation and invasion, and induces apoptosis in MDA-MB-231 cells. (A) The expression of miR-320 in MDA-MB-231 cells transfected with miR-320 mimics or NC. (B) Colony formation analysis of miR-320-overexpressing and NC MDA-MB-231 cells. (C) MTT analysis of miR-320-overexpressing and NC MDA-MB-231 cells. (D) The cell cycle distribution of miR-320-overexpressing and NC MDA-MB-231 cells. (E) Apoptosis analysis of miR-320-overexpressing and NC MDA-MB-231 cells. (F) Transwell invasion assay of miR-320-overexpressing and NC MDA-MB-231 cells (magnification, $x 400$ ). ${ }^{\# \#} \mathrm{P}<0.01$ and ${ }^{\# \# \#} \mathrm{P}<0.001$ vs. NC. miR, microRNA; NC, negative control; PI, propidium iodide; FITC, fluorescein isothiocyanate. 
A
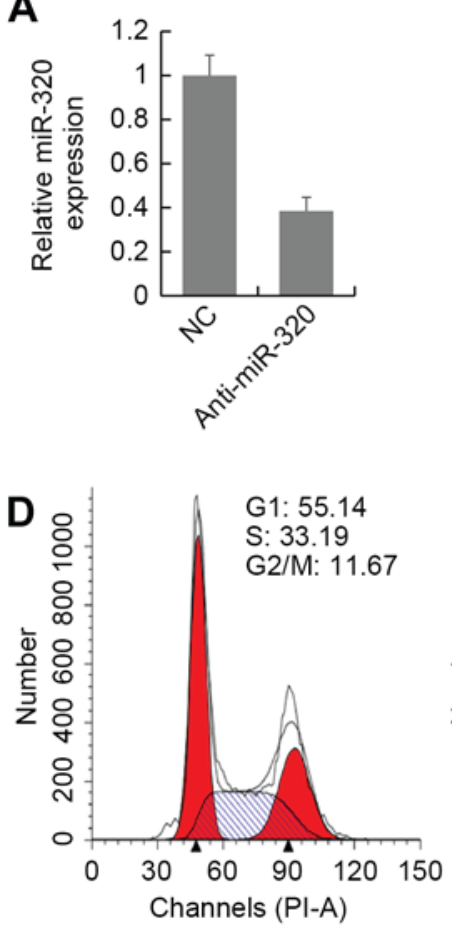

NC
B

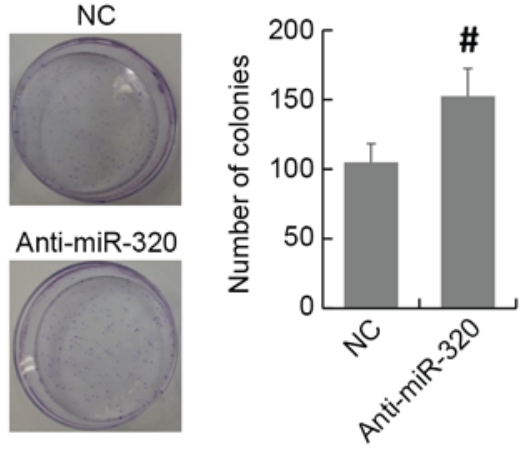

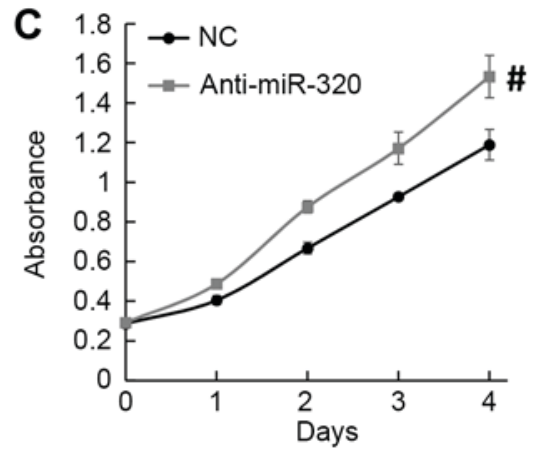

E
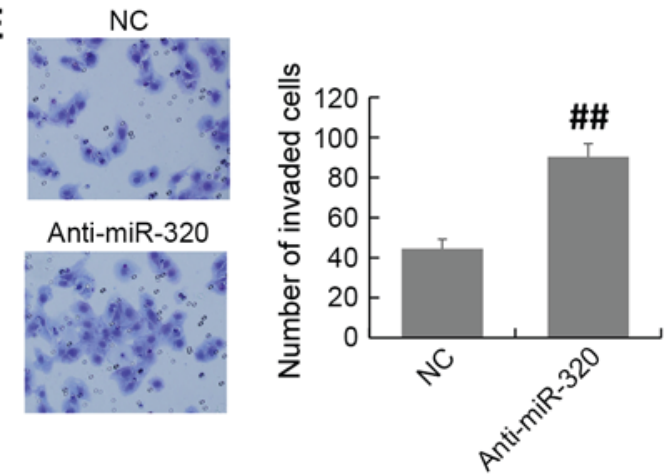

Figure 3. Knockdown of miR-320 promotes cell proliferation and invasion in MCF7 cells. (A) The expression of miR-320 in miR-320-depleted (anti-miR-320) and NC MCF7 cells. (B) Colony formation analysis of miR-320-depleted and NC MCF7 cells. (C) MTT analysis of miR-320-depleted and NC MCF7 cells. (D) Cell cycle distribution of miR-320-depleted and NC MCF7 cells. (E) Transwell analysis of miR-320-depleted and NC MCF7 cells (magnification, x400). ${ }^{\#} \mathrm{P}<0.05$ and ${ }^{\# \#} \mathrm{P}<0.01$ vs. NC. miR, microRNA; NC, negative control.

site-directed mutagenesis kit. The luciferase assay demonstrated that overexpression of miR-320 significantly decreased the luciferase activity of the SOX4-1wt and SOX4-2wt constructs relative to NC-transfected cells (Fig. 4B). Furthermore, mutation of the miR-320 binding sites prevented this effect of miR-320 on luciferase activity: There were no significant differences between the miR-320 mimic-transfected and the NC cells (Fig. 4C). Furthermore, RT-qPCR and western blot assays revealed that the overexpression of miR-320 downregulated the expression of SOX4 in MDA-MB-231 cells, whereas knockdown of miR-320 upregulated the expression of SOX4 in MCF7 cells (Fig. 4D and E). Together, these results suggested that SOX4 is a direct target of miR-320.

miR-320 inhibits breast cancer progression by downregulating SOX4. To additionally demonstrate the regulation of SOX4 by miR-320 in breast cancer cells, pcDNA3.1-SOX4 was transfected into the miR-320-overexpressed MDA-MB-231 cells to rescue the SOX4 expression. The RT-qPCR assay indicated that the expression of SOX4 was significantly increased in miR-320/SOX4 co-transfected MDA-MB-231 cells compared with that of NC cells and cells transfected with miR-320 alone (Fig. 5A). The colony formation, MTT and Transwell invasion assays demonstrated that overexpression of SOX 4 could rescue the malignant phenotype of miR-320-overexpressing MDA-MB-231 cells (Fig. 5B-D). Furthermore, the expression of epithelial marker E-cadherin was downregulated and the mesenchymal marker vimentin was upregulated in SOX4/miR-320-overexpressing MDA-MB-231 cells compared with miR-320-overexpressing cells (Fig. 5E). Therefore, the results suggested that miR-320 inhibited breast cancer progression by downregulating SOX4.

\section{Discussion}

miRNAs are critical regulators involved in a number of biological processes, including proliferation, differentiation, migration, metabolism and apoptosis (33). The abnormal expression of miRNAs has been observed in a number types of cancer $(34,35)$. Thus, the identification and study of cancer-specific miRNAs and their targets are critical for understanding their function and mechanism in cancer development and progression. miR-320 has been identified to be downregulated in a number of types of cancer, including cervical cancer, colon cancer, oral cancer and osteosarcoma, and was shown to be involved in tumorigenesis and progression as a tumor suppressor $(24,25,28,36)$. A miRNA microarray analysis demonstrated that miR-321 was downregulated in patients with breast cancer, suggesting that miR-320 may serve an important function in breast cancer development and progression (37). However, the molecular mechanism by which miR-320 affects breast cancer development and progression remains unknown. Therefore, the present study aimed to elucidate the biological function and mechanism of miR-320 in breast cancer.

Consistent with a previous study (38), the results of the present study indicated that miR-320 was decreased in the majority of breast cancer tissues compared with the corresponding adjacent normal tissues. Overexpression of miR-320 inhibited proliferation and invasion, and induced apoptosis, in breast cancer cells, suggesting that miR-320 

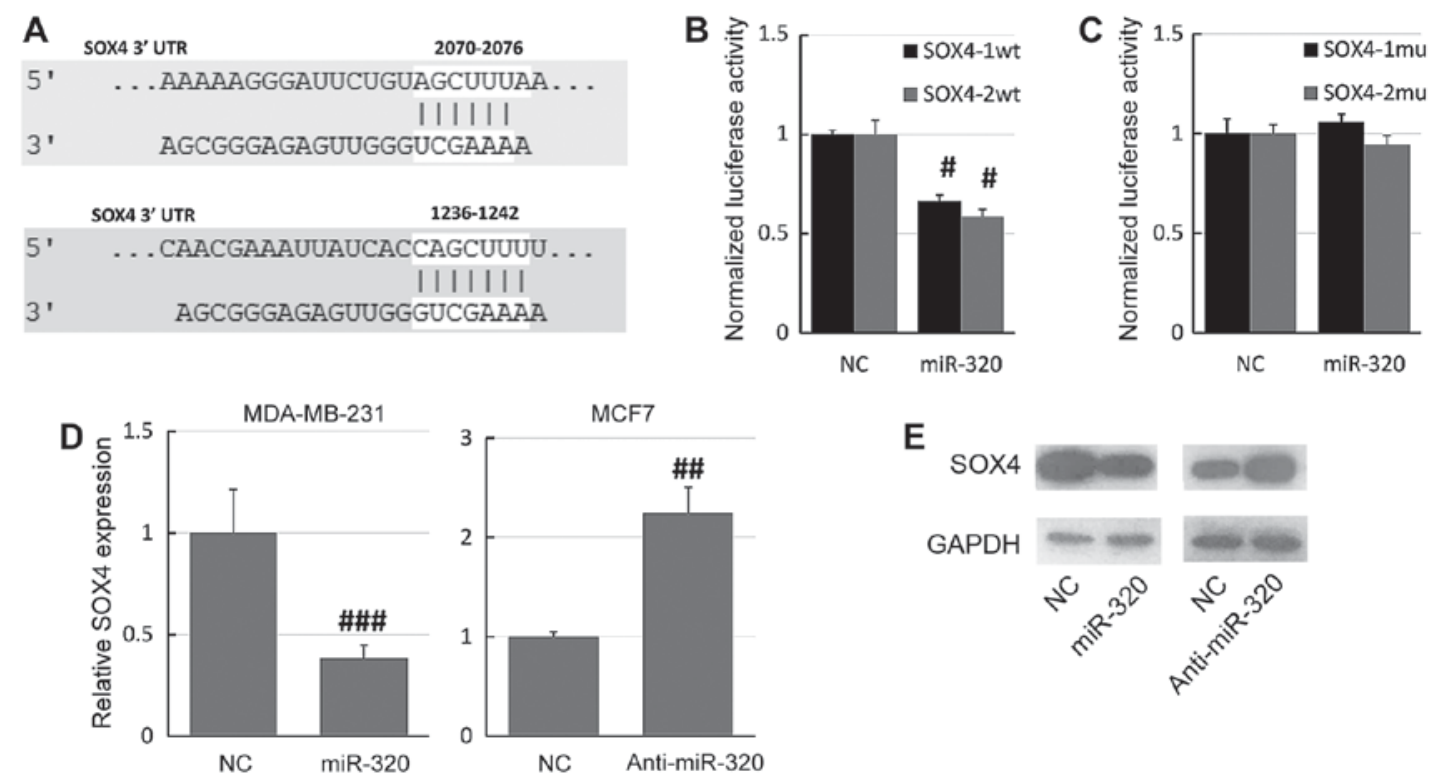

Figure 4. SOX4 is a direct target of miR-320. (A) Schematic representation of SOX4 3'-UTRs demonstrating the putative miR target site. The relative luciferase activity of (B) wt and (C) mu SOX4 3'-UTRs. The expression of SOX4 level in miR-320-overexpressing MDA-MB-231 and miR-320-depleted MCF7 cells, determined using (D) RT-qPCR and (E) western blot analysis. GAPDH was used as the loading control. ${ }^{\#} \mathrm{P}<0.05,{ }^{\# \#} \mathrm{P}<0.01$ and ${ }^{\# \# /} \mathrm{P}<0.001$ vs. corresponding NC group. SOX4, SRY-box 4; miR, microRNA; UTR, untranslated region; NC, negative control; wt, wild-type; mu, mutated.

A
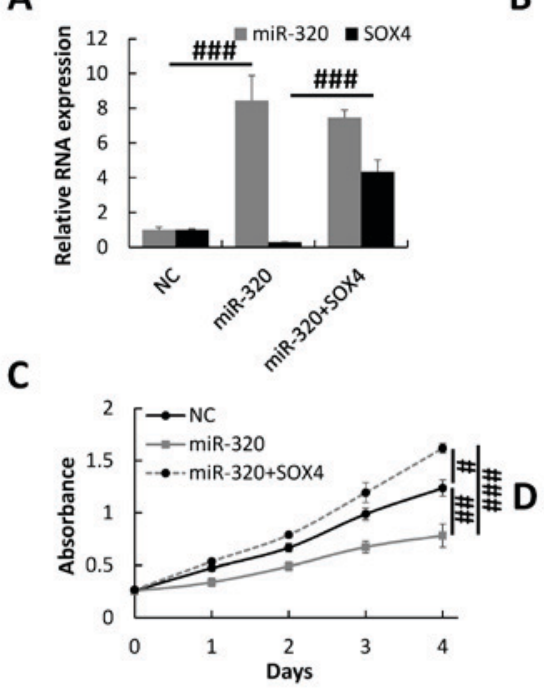

E

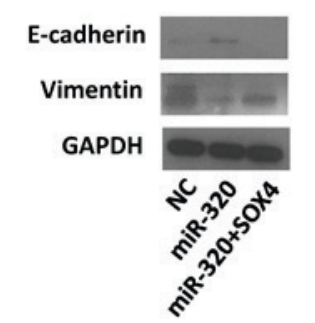

B
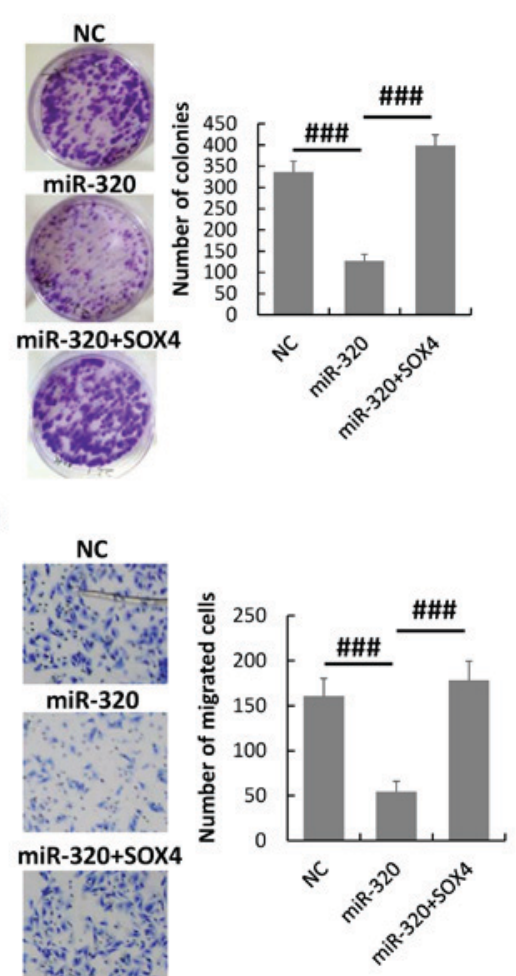

Figure 5. miR-320 inhibits breast cancer progression by downregulating SOX4. (A) Expression of miR-320 and SOX4 in MDA-MB-231 cells transfected with NC, miR-320 mimics alone, or miR-320 mimics plus SOX4. (B) Colony formation analysis of transfected cells. (C) MTT analysis of transfected cells. (D) Transwell invasion analysis of transfected cells (magnification, $\mathrm{x} 400$ ). (E) The expression of E-cadherin and vimentin in transfected cells. ${ }^{*} \mathrm{P}<0.05$, ${ }^{\# \#} \mathrm{P}<0.01,{ }^{\# \#} \mathrm{P}<0.001$ vs. NC. SOX4, SRY-box 4; miR, microRNA; NC, negative control.

may be a tumor suppressor and a diagnostic biomarker in breast cancer. In addition, the present study demonstrated a potential underlying molecular mechanism of miR-320 in the inhibition of breast cancer progression; the results indicated that SOX4 is a direct target of miR-320 in breast cancer, which is consistent with the mechanism of miR-320/SOX4 in 
colorectal cancer (29). Furthermore, it was demonstrated that the inhibitory effects of miR-320 overexpression on breast cancer cell proliferation and invasion were partially attenuated by the upregulation of SOX4 expression. Thus, it was indicated that miR-320 inhibits breast cancer progression by targeting SOX4.

EMT is a critical process in breast cancer progression that causes epithelial cells to acquire fibroblast-like properties, with reduced intercellular adhesion and increased motility (20). SOX4 has been observed to be overexpressed in a number of different types of malignant tumor and is one of the master regulators in EMT-induced cancer metastasis and chemoresistance $(5,10,39,40)$. The results of the present study demonstrated that miR-320 inhibits the EMT in breast cancer cells, as demonstrated by the retention of the epithelial phenotype, and the EMT-inhibiting effect of miR-320 may be attenuated by the overexpression of SOX4. Thus, to the best of our knowledge, the current study is the first to demonstrate that miR-320 is a regulator of SOX4 in breast cancer cells, which provides one possible mechanism underlying the function of miR-320 in breast cancer progression.

In summary, the results of the present study indicated that miR-320 is frequently decreased in breast cancer cells and that miR-320 inhibits breast cancer progression by targeting SOX4. This novel miR-320/SOX4 axis may provide insight into the molecular mechanisms underlying breast cancer progression, and the upregulation of miR-320 expression may be a therapeutic strategy for the treatment of breast cancer.

\section{References}

1. Siegel RL, Miller KD and Jemal A: Cancer statistics, 2016. CA Cancer J Clin 66: 7-30, 2016.

2. Karlsson MC, Gonzalez SF, Welin $J$ and Fuxe J: Epithelial-mesenchymal transition in cancer metastasis through the lymphatic system. Mol Oncol 11: 781-791, 2017.

3. Poncy A, Antoniou A, Cordi S, Pierreux CE, Jacquemin P and Lemaigre FP: Transcription factors SOX4 and SOX9 cooperatively control development of bile ducts. Dev Biol 404: 136-148, 2015.

4. Jang SM, Kim JW, Kim CH, An JH, Johnson A, Song PI, Rhee S and Choi KH: KAT5-mediated SOX4 acetylation orchestrates chromatin remodeling during myoblast differentiation. Cell Death Dis 6: e1857, 2015.

5. Tiwari N, Tiwari VK, Waldmeier L, Balwierz PJ, Arnold P, Pachkov M, Meyer-Schaller N, Schübeler D, van Nimwegen E and Christofori G: Sox 4 is a master regulator of epithelial-mesenchymal transition by controlling Ezh2 expression and epigenetic reprogramming. Cancer Cell 23: 768-783, 2013.

6. Wang B, Li Y, Tan F and Xiao Z: Increased expression of SOX4 is associated with colorectal cancer progression. Tumour Biol 37: 9131-9137, 2016.

7. Bilir B, Osunkoya AO, Wiles WG IV, Sannigrahi S, Lefebvre V, Metzger D, Spyropoulos DD, Martin WD and Moreno CS: SOX4 is essential for prostate tumorigenesis initiated by PTEN ablation. Cancer Res 76: 1112-1121, 2016.

8. Hasegawa S, Nagano H, Konno M, Eguchi H, Tomokuni A, Tomimaru Y, Asaoka T, Wada H, Hama N, Kawamoto K, et al: A crucial epithelial to mesenchymal transition regulator, Sox4/Ezh2 axis is closely related to the clinical outcome in pancreatic cancer patients. Int J Oncol 48: 145-152, 2016.

9. Sung WJ, Kim H and Park KK: The biological role of epithelial-mesenchymal transition in lung cancer (Review). Oncol Rep 36: 1199-1206, 2016.

10. Zhang J, Liang Q, Lei Y, Yao M, Li L, Gao X, Feng J, Zhang Y, Gao H, Liu DX, et al: SOX4 induces epithelial-mesenchymal transition and contributes to breast cancer progression. Cancer Res 72: 4597-4608, 2012.
11. Sinner D, Kordich JJ, Spence JR, Opoka R, Rankin S, Lin SC, Jonatan D, Zorn AM and Wells JM: Sox17 and Sox4 differentially regulate beta-catenin/T-cell factor activity and proliferation of colon carcinoma cells. Mol Cell Biol 27: 7802-7815, 2007.

12. Jiao C, Song Z, Chen J, Zhong J, Cai W, Tian S, Chen S, Yi Y and Xiao Y: lncRNA-UCA1 enhances cell proliferation through functioning as a ceRNA of Sox4 in esophageal cancer. Oncol Rep 36: 2960-2966, 2016.

13. Wang W, Zhang E and Lin C: MicroRNAs in tumor angiogenesis. Life Sci 136: 28-35, 2015.

14. Huang J, Zhang SY, Gao YM, Liu YF, Liu YB, Zhao ZG and Yang K: MicroRNAs as oncogenes or tumour suppressors in oesophageal cancer: Potential biomarkers and therapeutic targets. Cell Prolif 47: 277-286, 2014.

15. Kim VN, Han J and Siomi MC: Biogenesis of small RNAs in animals. Nat Rev Mol Cell Biol 10: 126-139, 2009.

16. Thomson DW, Bracken CP and Goodall GJ: Experimental strategies for microRNA target identification. Nucleic Acids Res 39: 6845-6853, 2011

17. Rupaimoole R, Calin GA, Lopez-Berestein G and Sood AK: miRNA deregulation in cancer cells and the tumor microenvironment. Cancer Discov 6: 235-246, 2016.

18. Magee P, Shi L and Garofalo M: Role of microRNAs in chemoresistance. Ann Transl Med 3: 332, 2015.

19. Yin K, Yin W, Wang Y, Zhou L, Liu Y, Yang G, Wang J and Lu J: MiR-206 suppresses epithelial mesenchymal transition by targeting TGF- $\beta$ signaling in estrogen receptor positive breast cancer cells. Oncotarget 7: 24537-24548, 2016.

20. Yu Y, Zhao Y, Sun XH, Ge J, Zhang B, Wang X and Cao XC: Down-regulation of miR-129-5p via the Twist1-Snail feedback loop stimulates the epithelial-mesenchymal transition and is associated with poor prognosis in breast cancer. Oncotarget 6: 34423-34436, 2015.

21. Rhodes LV, Martin EC, Segar HC, Miller DF, Buechlein A, Rusch DB, Nephew KP, Burow ME and Collins-Burow BM: Dual regulation by microRNA-200b-3p and microRNA-200b-5p in the inhibition of epithelial-to-mesenchymal transitionintriple-negative breast cancer. Oncotarget 6: 16638-16652, 2015.

22. Yang S, Li Y, Gao J, Zhang T, Li S, Luo A, Chen H, Ding F, Wang X and Liu Z: MicroRNA-34 suppresses breast cancer invasion and metastasis by directly targeting Fra-1. Oncogene 32: 4294-4303, 2013

23. Pichler M and Calin GA: MicroRNAs in cancer: From developmental genes in worms to their clinical application in patients. $\mathrm{Br}$ J Cancer 113: 569-573, 2015.

24. Wan LY, Deng J, Xiang XJ, Zhang L, Yu F, Chen J, Sun Z, Feng $M$ and Xiong JP: miR-320 enhances the sensitivity of human colon cancer cells to chemoradiotherapy in vitro by targeting FOXM1. Biochem Biophys Res Commun 457: $125-132,2015$.

25. Zhang T, Zou P, Wang T, Xiang J, Cheng J, Chen D and Zhou J: Down-regulation of miR-320 associated with cancer progression and cell apoptosis via targeting Mcl-1 in cervical cancer. Tumour Biol 37: 8931-8940, 2016.

26. Sun JY, Xiao WZ, Wang F, Wang YQ, Zhu YH, Wu YF, Miao ZL and Lin YC: MicroRNA-320 inhibits cell proliferation in glioma by targeting E2F1. Mol Med Rep 12: 2355-2359, 2015.

27. Hsieh IS, Chang KC, Tsai YT, Ke JY, Lu PJ, Lee KH, Yeh SD, Hong TM and Chen YL: MicroRNA-320 suppresses the stem cell-like characteristics of prostate cancer cells by downregulating the Wnt/beta-catenin signaling pathway. Carcinogenesis 34: 530-538, 2013.

28. Wu YY, Chen YL, Jao YC, Hsieh IS, Chang KC and Hong TM: miR-320 regulates tumor angiogenesis driven by vascular endothelial cells in oral cancer by silencing neuropilin 1 . Angiogenesis 17: 247-260, 2014

29. Vishnubalaji R, Hamam R, Yue S, Al-Obeed O, Kassem M, Liu FF, Aldahmash A and Alajez NM: MicroRNA-320 suppresses colorectal cancer by targeting SOX4, FOXM1, and FOXQ1. Oncotarget 7: 35789-35802, 2016.

30. Lakhani SR, Ellis IO, Schnitt SJ, Tan PH and van de Vijver MJ (eds): WHO Classification of Tumours of the Breast 4th edition. Lyon, IARC Press, 2012.

31. Zhang YF, Yu Y, Song WZ, Zhang RM, Jin S, Bai JW, Kang HB, Wang X and Cao XC: miR-410-3p suppresses breast cancer progression by targeting Snail. Oncol Rep 36: 480-486, 2016.

32. Betel D, Wilson M, Gabow A, Marks DS and Sander C: The microRNA.org resource: Targets and expression. Nucleic Acids Res 36 (Database Issue): D149-D153, 2008. 
33. Adlakha YK and Saini N: MicroRNA: A connecting road between apoptosis and cholesterol metabolism. Tumour Biol 37: 8529-8554, 2016.

34. Chen Y, Yang X, Xu Y, Cao J and Chen L: Genomic analysis of drug resistant small cell lung cancer cell lines by combining mRNA and miRNA expression profiling. Oncol Lett 13: 4077-4084, 2017.

35. MacedoT, Silva-Oliveira RJ, Silva VAO, VidalDO, Evangelista AF and Marques MMC: Overexpression of mir-183 and mir-494 promotes proliferation and migration in human breast cancer cell lines. Oncol Lett 14: 1054-1060, 2017.

36. Cheng C, Chen ZQ and Shi XT: MicroRNA-320 inhibits osteosarcoma cells proliferation by directly targeting fatty acid synthase. Tumour Biol 35: 4177-4183, 2014
37. Yan LX, Huang XF, Shao Q, Huang MY, Deng L, Wu QL, Zeng YX and Shao JY: MicroRNA miR-21 overexpression in human breast cancer is associated with advanced clinical stage, lymph node metastasis and patient poor prognosis. RNA 14: 2348-2360, 2008.

38. Wang B, Yang Z, Wang H, Cao Z, Zhao Y, Gong C, Ma L, Wang X, Hu X and Chen S: MicroRNA-320a inhibits proliferation and invasion of breast cancer cells by targeting RAB11A. Am J Cancer Res 5: 2719-2729, 2015.

39. Jin Y,ZhaoM,XieQ,Zhang H,Wang QandMaQ: MicroRNA-338-3p functions as tumor suppressor in breast cancer by targeting SOX4. Int J Oncol 47: 1594-1602, 2015.

40. Liu Y, Li Y, Liu J, Wu Y and Zhu Q: MicroRNA-132 inhibits cell growth and metastasis in osteosarcoma cell lines possibly by targeting Sox4. Int J Oncol 47: 1672-1684, 2015. 